\title{
Supplementary material to \\ 'Global application of a surface mass balance model using gridded climate data'
}

\author{
R. H. Giesen and J. Oerlemans
}

This supplementary material contains information on the 80 glaciers that were used to examine the applicability of the mass balance model in different regions. We selected all glaciers for which both winter and annual mass balance profiles were available from the World Glacier Monitoring Service (WGMS). At the time the data was requested from WGMS (June 2010), the most recent year with available mass balance measurements was 2008. The characteristics and parameter values listed for all 80 glaciers are described in Table 1, the list of glaciers is given in Table 2.

Table 1: Description of variables listed for the 80 glaciers in Table 2.

\begin{tabular}{|c|c|c|}
\hline Variable & Unit & Description \\
\hline lat & ${ }^{\circ} \mathrm{N}$ & glacier latitude \\
\hline lon & ${ }^{\circ} \mathrm{E}$ & glacier longitude \\
\hline \# & & number of years with both winter and annual mass balance profiles \\
\hline period & & period of calendar years spanned by the available profiles \\
\hline ELA & m a.s.l. & equilibrium-line altitude \\
\hline$z_{\mathrm{CRU}}$ & m a.s.l. & elevation of the nearest CRU climate data gridpoint \\
\hline$\gamma$ & $\mathrm{mm} \mathrm{a}^{-1} \mathrm{~m}^{-1}$ & vertical precipitation gradient \\
\hline$p$ & & precipitation multiplication factor \\
\hline$\tau$ & & atmospheric transmissivity \\
\hline$\psi_{\min }$ & $\mathrm{W} \mathrm{m}^{-2}$ & minimum temperature-dependent flux \\
\hline$c$ & $\mathrm{~W} \mathrm{~m}^{-2} \mathrm{~K}^{-1}$ & slope of the temperature-dependent flux \\
\hline$T_{\text {tip }}$ & ${ }^{\circ} \mathrm{C}$ & tipping point in the temperature-dependent flux equation \\
\hline$T_{\text {corr }}$ & ${ }^{\circ} \mathrm{C}$ & air temperature correction \\
\hline$C_{T}$ & m w.e. $\mathrm{K}^{-1}$ & mass balance sensitivity to changes in temperature \\
\hline$C_{P}$ & m w.e. $[10 \%]^{-1}$ & mass balance sensitivity to changes in precipitation \\
\hline$C_{\tau}$ & m w.e. $[0.05]^{-1}$ & mass balance sensitivity to changes in atmospheric transmissivity \\
\hline
\end{tabular}


Table 2: The 80 glaciers with winter and annual mass balance profiles available from WGMS. Given are the glacier name and all attributes described in Table 1. The values for $\gamma, p, C_{T}, C_{P}$ and $C_{\tau}$ are the median values for the three calibration cases $\tau$-cal., $T$-cal. and $\psi$-cal. The AWS parameter set used in combination with the calibrated parameters is given in brackets after the region name. This parameter set is used as a basis, combined with calibrated values for either $\tau$ (case $\tau$-cal.), the parameters in the temperature-dependent flux $\psi_{\min }, c$ and $T_{\text {tip }}$ (case $\psi$-cal.) or the temperature correction $T_{\text {corr }}$ (case $T$-cal.).

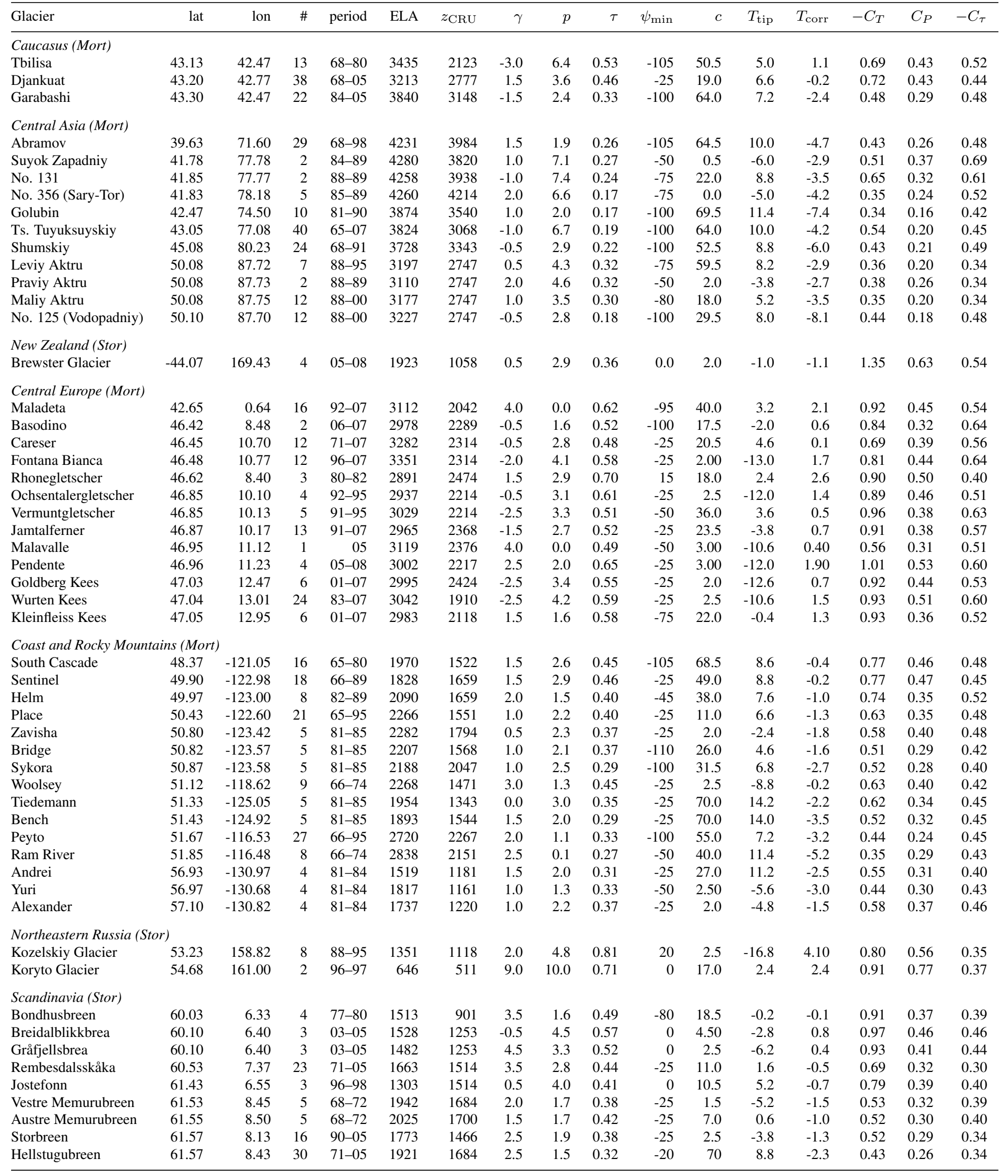


Table 2: (continued)

\begin{tabular}{|c|c|c|c|c|c|c|c|c|c|c|c|c|c|c|c|c|}
\hline Glacier & lat & lon & $\#$ & period & ELA & $z_{\mathrm{CRU}}$ & $\gamma$ & $p$ & $\tau$ & $\psi_{\min }$ & $c$ & $T_{\mathrm{tip}}$ & $T_{\text {corr }}$ & $-C_{T}$ & $C_{P}$ & $-C_{\tau}$ \\
\hline Tunsbergdalsbreen & 61.60 & 7.05 & 7 & $66-72$ & 1449 & 1250 & 2.0 & 2.5 & 0.35 & -25 & 6.5 & 2.0 & -1.7 & 0.57 & 0.31 & 0.35 \\
\hline Spørteggbreen & 61.61 & 7.47 & 1 & 91 & 1528 & 1298 & 2.0 & 1.5 & 0.21 & -50 & 1.5 & -2.6 & -3.6 & 0.45 & 0.24 & 0.32 \\
\hline Gråsubreen & 61.65 & 8.60 & 30 & $71-05$ & 2147 & 1700 & -2.0 & 2.0 & 0.31 & -75 & 35.5 & 2.8 & -3.2 & 0.54 & 0.23 & 0.41 \\
\hline Nigardsbreen & 61.72 & 7.13 & 32 & $71-07$ & 1507 & 1396 & 2.0 & 2.5 & 0.40 & -25 & 21.0 & 4.6 & -0.7 & 0.66 & 0.33 & 0.31 \\
\hline Ålfotbreen & 61.75 & 5.65 & 36 & $65-05$ & 1163 & 727 & -0.5 & 3.1 & 0.59 & 0 & 5.0 & -2.0 & 1.0 & 1.05 & 0.53 & 0.37 \\
\hline Hansebreen & 61.75 & 5.68 & 11 & $91-05$ & 1178 & 807 & 3.0 & 2.5 & 0.55 & 0 & 7.5 & 0.6 & 0.7 & 1.07 & 0.52 & 0.42 \\
\hline Austdalsbreen & 61.80 & 7.35 & 13 & $88-05$ & 1478 & 1330 & 5.0 & 2.6 & 0.34 & -25 & 67.5 & 7.8 & -1.4 & 0.69 & 0.32 & 0.34 \\
\hline Okstindbreen & 66.23 & 14.37 & 7 & $90-96$ & 1345 & 556 & 3.5 & 0.5 & 0.49 & -105 & 20.5 & -1.6 & 0.1 & 0.59 & 0.32 & 0.30 \\
\hline Høgtuvbreen & 66.45 & 13.65 & 7 & $71-77$ & 847 & 653 & 3.0 & 2.4 & 0.46 & 0 & 11.5 & 4.0 & -0.2 & 0.82 & 0.43 & 0.36 \\
\hline Svartisheibreen & 66.55 & 13.77 & 4 & $91-94$ & 975 & 866 & 1.5 & 2.5 & 0.41 & -25 & 65.0 & 7.0 & -0.8 & 0.74 & 0.38 & 0.31 \\
\hline Engabreen & 66.65 & 13.85 & 30 & $71-05$ & 1095 & 838 & 5.0 & 1.8 & 0.49 & 0 & 14.5 & 4.6 & 0.1 & 0.73 & 0.40 & 0.30 \\
\hline Storglombreen & 66.67 & 14.00 & 5 & $88-05$ & 1274 & 707 & 6.0 & 0.7 & 0.52 & 0 & 46.5 & 6.2 & 0.4 & 0.78 & 0.37 & 0.37 \\
\hline Trollbergdalsbreen & 66.72 & 14.45 & 10 & $70-94$ & 1094 & 987 & 2.5 & 2.5 & 0.46 & 0 & 20.5 & 5.6 & -0.3 & 0.77 & 0.37 & 0.37 \\
\hline Partejekna & 67.17 & 17.67 & 4 & $97-00$ & 1703 & 1137 & 1.0 & 4.0 & 0.40 & -25 & 2.0 & -9.4 & -0.9 & 0.58 & 0.30 & 0.34 \\
\hline Rabots Glaciär & 67.90 & 18.55 & 15 & $86-06$ & 1442 & 1245 & 5.5 & 3.0 & 0.36 & -25 & 1.5 & -8.2 & -1.5 & 0.48 & 0.26 & 0.32 \\
\hline Storglaciären & 67.90 & 18.57 & 32 & $71-07$ & 1486 & 1245 & 10 & 2.0 & 0.42 & -25 & 2.5 & -8.2 & -0.4 & 0.52 & 0.30 & 0.31 \\
\hline Tarfalaglaciären & 67.93 & 18.65 & 7 & $00-07$ & 1514 & 1245 & 0.5 & 4.5 & 0.52 & 0 & 1.5 & -5.2 & 0.4 & 0.65 & 0.36 & 0.36 \\
\hline Riukojietna & 68.08 & 18.08 & 10 & $96-07$ & 1381 & 1153 & -0.5 & 3.4 & 0.43 & -25 & 32.0 & 5.2 & -0.6 & 0.63 & 0.26 & 0.37 \\
\hline Cainhavarre & 68.10 & 18.00 & 4 & $65-68$ & 1347 & 1153 & 0.0 & 3.7 & 0.39 & -25 & 2.0 & -7.8 & -1.0 & 0.58 & 0.30 & 0.33 \\
\hline Storsteinsfjellbreen & 68.22 & 17.92 & 9 & $65-95$ & 1317 & 1019 & 4.0 & 2.0 & 0.37 & -25 & 1.5 & -9.2 & -1.1 & 0.49 & 0.27 & 0.27 \\
\hline Blåisen & 68.33 & 17.85 & 4 & $65-68$ & 1063 & 1019 & 6.5 & 3.6 & 0.27 & -25 & 1.5 & -1.8 & -2.4 & 0.50 & 0.27 & 0.33 \\
\hline Marmaglaciären & 68.83 & 18.67 & 14 & $95-08$ & 1616 & 749 & 2.5 & 0.0 & 0.48 & -25 & 5.5 & -3.0 & 0.0 & 0.48 & 0.27 & 0.35 \\
\hline Langfjordjøkelen & 70.12 & 21.77 & 9 & $91-05$ & 889 & 548 & 2.5 & 4.6 & 0.50 & 0 & 6.0 & 1.0 & 0.2 & 0.74 & 0.34 & 0.35 \\
\hline \multicolumn{17}{|l|}{ Arctic (Kong) } \\
\hline Devon Ice Cap & 75.42 & -83.25 & 3 & $96-98$ & 1125 & 1489 & 0.0 & 0.8 & 0.20 & -75 & 0.0 & -11.4 & -7.3 & 0.16 & 0.06 & 0.16 \\
\hline Hansbreen & 77.08 & 15.67 & 15 & $91-07$ & 364 & 299 & 2.5 & 4.2 & 0.35 & -30 & 0.0 & -9.0 & -1.8 & 0.53 & 0.20 & 0.26 \\
\hline Austre Brøggerbreen & 78.88 & 11.83 & 5 & $90-95$ & 428 & 215 & 1.5 & 2.1 & 0.38 & -30 & 40.0 & 6.8 & -1.6 & 0.51 & 0.18 & 0.27 \\
\hline
\end{tabular}

